\title{
Modelización del ensayo de resistencia a compresión del concreto de alta resistencia mediante una red neuronal artificial. Obtención de la incertidumbre del resultado
}

\section{Modeling the resistance to compression of high performance concrete test by means of an artificial neural network. Obtaining the uncertainty of the results}

\author{
Francisco García Fernández¹, Ana Torre Carrillo², Isabel Moromi Nakata², Pedro Espinoza Haro ${ }^{3}$ y Luis Acuña \\ Pinaud $^{3}$ \\ ${ }^{1}$ Dpto. Sistemas y Recursos Naturales. Universidad Politécnica de Madrid. Ciudad Universitaria S/N, 28040 \\ Madrid, España \\ ${ }^{2}$ Facultad de Ingeniería Civil, Universidad Nacional de Ingeniería. Av. Túpac Amaru, 210. Lima 25, Perú \\ ${ }^{3}$ Facultad de Ingeniería Industrial y de Sistemas. Universidad Nacional de Ingeniería. Av. Túpac Amaru, 210. \\ Lima 25, Perú
}

DOI: https://doi.org/10.33017/RevECIPeru2015.0012/

\section{Resumen}

En los últimos años las ANN han tenido un gran desarrollo en el control de procesos industriales debido principalmente a su capacidad de modelizar relaciones complejas, que los sistemas tradicionales no han sido capaces de hacer, y predecir satisfactoriamente si las características de un producto se adecuan o no a las especificaciones correspondientes. Estas estructuras han sido ampliamente utilizadas en la caracterización de otros materiales como cemento, hormigón, algunos metales o la madera.

El perceptrón multicapa, una de las redes neuronales artificiales más populares, se ha convertido en los últimos tiempos en una potente herramienta de modelización en numerosos campos que van desde las finanzas, a la ingeniería o la medicina. Esta herramienta consigue mejorar sustancialmente cualquier modelo previo propuesto para modelizar cualquier sistema independientemente de la naturaleza de éste, con la ventaja añadida de que no necesitan ninguna suposición previa sobre la estructura de los datos

Sin embargo, la red sólo proporciona el valor de la salida sin ninguna información acerca de su precisión. La obtención de la incertidumbre de salida es importante, no sólo porque proporciona un intervalo de confianza sobre el valor de salida, sino porque da una idea de la calidad del método de medida. Esta incertidumbre procede de dos fuentes, por un lado el ruido inherente a los valores de entrada y por otro la simplificación del fenómeno que todo modelo matemático supone.

En este trabajo se va a desarrollar una nueva metodología para obtener tanto la incertidumbre como los intervalos de confianza de la salida de un modelo específico de red neuronal, el perceptrón multicapa, basándose en el método de simulación de Montecarlo especificado en Suplemento 1 de la GUM para posteriormente aplicarlo a la modelizacion del ensayo de resistencia a compression del concreto.

Descriptores: Concreto de alta resistencia, red neuronal artificial, resistencia a compresión, incertidumbre, Método de Monte Carlo

\section{Abstract}

Major advances have been made with the use of ANNs in recent years in industrial process control, mainly because they are capable of modeling complex relations, unlike conventional systems, and can adequately predict whether or not the characteristics of a product are in line with specifications. They have been widely used to characterize other materials such as cement, concrete, certain metals or wood. 
The multilayer perceptron, one of the most popular artificial neural networks, has become a powerful modeling tool in numerous fields, ranging from finances to engineering and medicine. This tool is capable of considerably improving on all previous models proposed for modeling any system, regardless of its nature, with the added advantage that no prior assumption on the structure of the data is necessary.

However, the network provides only the output value, with no information about its accuracy. Obtaining the output uncertainty is important, not only because it provides a coverage interval for the output value, but also because it indicates the quality of the measuring method. This uncertainty comes from two sources: firstly, the inherent uncertainty in the input data, and secondly, the simplification of the phenomenon involved in any mathematical model.

This study develops a new methodology for obtaining both the output uncertainty and coverage intervals of a specific neural network model - the multilayer perceptron - based on the Monte Carlo simulation method indicated in Supplement 1 to the Guide to the Expression of Uncertainty in Measurement (GUM), in order to use it when modelling the test of resistance to compression of concrete.

Keywords: High performance concrete, artificial neural network, resistance to compression, uncertainty, Monte Carlo method

\section{Introducción}

En los últimos años, el desarrollo de ordenadores cada vez más potentes ha contribuido al aumento del uso de las técnicas de modelización mediante redes neuronales artificiales en muy diferentes ámbitos de la ingeniería y la investigación. Estas herramientas consiguen mejorar sustancialmente cualquier modelo previo propuesto con la ventaja añadida de que no necesitan ninguna suposición previa sobre la estructura estadística de los datos [1].

Sin embargo la red neuronal no proporciona ninguna información sobre los intervalos de confianza o la incertidumbre del valor de salida [1]. Esta incertidumbre es importante no sólo porque es indicativa de la calidad de nuestro proceso de medida, sino porque a la vez nos proporciona un intervalo de confianza sobre nuestro resultado $[2,3]$.

Según la definición, aceptada internacionalmente, la incertidumbre asociada a una medida se puede definir como la raíz cuadrada de la varianza de su función de densidad de probabilidad. La Guía de Expresión de la Incertidumbre del Resultado de Medidas (GUM) [4] explica un método para obtener la incertidumbre de una medida partiendo de los valores de los parámetros de entrada y de sus distribuciones de probabilidad y válido en la mayoría de los casos. Sin embargo, en algunas ocasiones en las que el mensurando se define como una función dependiente de los valores de entrada, implica el conocimiento de la función que relaciona los parámetros de entrada con el mensurando, lo cual se encuentra con los problemas de modelos especificados por soluciones numéricas como es el caso de modelos definidos mediante ecuaciones diferenciales [5] o excesivamente complejos y no lineales, como puede ser el caso de una red neuronal artificial. Esto hace que la evaluación de la incertidumbre de salida basada en la ley de propagación de las incertidumbres sea de imposible aplicación o proporcione unos valores no del todo fiables [5].

Para solucionar estos problemas el Grupo de Trabajo del Joint Commitee for Guides in Metrology (JCGM) elaboró un suplemento, válido para un grupo mayor de situaciones [6], dónde se detalla la obtención de la incertidumbre de salida del mensurando a partir de la simulación por el Método de Montecarlo (MCM) [7].

En este artículo se va a desarrollar una nueva metodología, basándose en el método de simulación de Montecarlo especificado en Suplemento 1 de la GUM [7], para obtener la incertidumbre de la salida de un perceptrón multicapa aplicado a la modelización del ensayo de resistencia a compresión del concreto de alta resistencia de acuerdo a la norma ASTM C39/C 39M [8] tras diferentes periodos de curado.

\section{Material y Métodos}

\subsection{Concreto de alta resistencia.}

En este estudio se han utilizado 1054 probetas de concreto de alta resistencia fabricadas con distintos tipos y cantidades de cemento, arena, piedra y agua. Las probetas para el ensayo de compresión se han fabricado de acuerdo a la norma ASTM C 192/C 192M [9]. Los ensayos de compresión se han realizado según la norma ASTM C39/C 39M [8] tras diferentes periodos de curado. 
Los ensayos de compresión axial se realizaron en una máquina TONI-TECHNIK provista de una célula de $3.000 \mathrm{KN}$ y TINIUS OLSEN DE $1.500 \mathrm{KN}$. De acuerdo a estudios similares realizados anteriormente [10-12]. Como variables explicativas se tomaron el tiempo de curado, tipo cantidad y porcentaje de aditivo; tipo, porcentaje y cantidad de microsílice; cantidades de agua, piedra, arena y cemento; el TNM de la piedra, los pesos específicos de la arena y la piedra y la relación agua-cemento.

La siguiente tabla (tabla 1) muestra los equipos utilizados en los ensayos así cómo su rango de media e incertidumbre.

Tabla 1. Equipos utilizados.

\begin{tabular}{lc}
\multicolumn{1}{c}{ Equipo } & Incertidumbre \\
\hline Tamiz 3/4" & $0.05 \mathrm{~mm}$ \\
Tamiz 1" & $0.04 \mathrm{~mm}$ \\
Balanza $(0-100) \mathrm{kg}$ & $0.02 \mathrm{~kg}$ \\
Balanza $(0-21000) \mathrm{g}$ & $2.5 \mathrm{~g}$ \\
Pie de rey $(0-300) \mathrm{mm}$ & $5 \cdot 10^{-3} \mathrm{~mm}$ \\
\hline
\end{tabular}

\subsection{El perceptrón multicapa}

El perceptrón multicapa (Fig. 1) lo podríamos definir como un sistema computacional que imita las capacidades computacionales de los sistemas biológicos utilizando un gran número de elementos interconectados. Su carácter de aproximador universal de funciones [13] le permite modelizar complejas relaciones no lineales.

El diseño de perceptrón multicapa es un proceso lento y complejo en el cual no existe una regla definida para determinar la estructura interna de la red. Sin embargo existen una serie de recomendaciones para su diseño, basadas en el número de datos disponibles [14] o en el tipo de configuración más deseable [15]. A esto hay que añadirle a una serie de condiciones que se deben cumplir para que la red cumpla su función con las garantías suficientes, entre ellas evitar el sobreentrenamiento [16].

Como función de transferencia se ha utilizado la tangente hiperbólica sigmoidea y todos los valores de las variables, tanto las independientes como la dependiente, se normalizaron mejorar la efectividad de la función de transferencia.
Como algoritmo de entrenamiento se ha utilizado la retropropagación resiliente, el cuál mejora notablemente los resultados para el caso de funciones de transferencia sigmoidea [16].

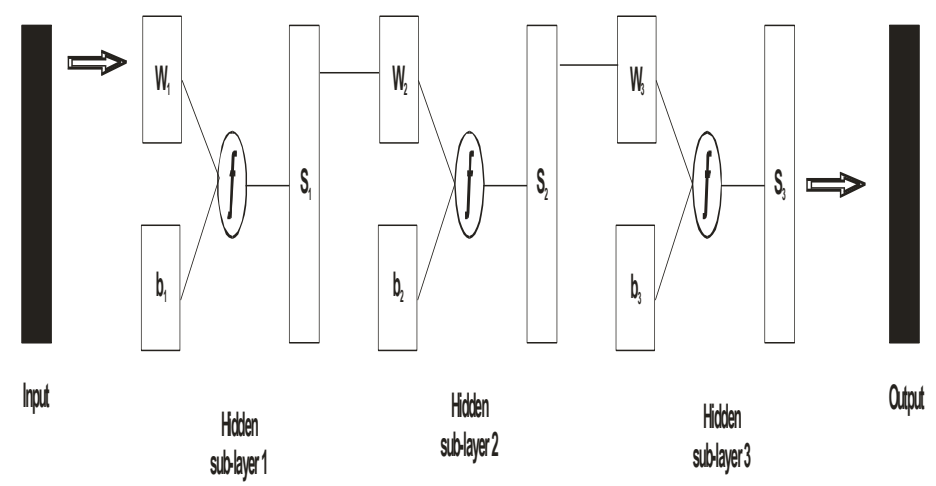

Fig. 1. Feed-forward multilayer perceptron neural network

\subsection{Método de Monte Carlo para el cálculo de la incertidumbre en los ensayos (método adaptativo).}

El Suplemento 1 de la GUM [8] describe el método numérico basado en la simulación de Monte Carlo para obtener dicha incertidumbre. El número de simulaciones dependerá del grado de tolerancia que se desee para el resultado final. Por regla general para unos intervalos del $95 \%$ de cobertura suelen ser necesarias unas $10^{6}$ simulaciones $[3,8]$.

El método adaptativo de Monte Carlo descrito en el apartado 7.9 del Suplemento 1 de la GUM [8] intenta reducir este número de simulaciones al definirlas a partir de un método iterativo basado en el nivel de precisión deseado para la incertidumbre y el factor de cobertura requerido.

El gráfico (Fig. 2) describe el proceso completo de obtención tanto de la red neuronal cómo de la incertidumbre de los datos de salida de un perceptrón multicapa.

Para evaluar la calidad de los intervalos de confianza se va a utilizar la probabilidad de cobertura de los intervalos de predicción (Prediction intervals coverage probability - PICP) $[1,17,18]$ (EC. 13) la cuál evalúa el número de datos experimentales incluidos dentro del intervalo de confianza. Esta medida es un buen indicador de la calidad de los intervalos de confianza obtenidos [1]. 


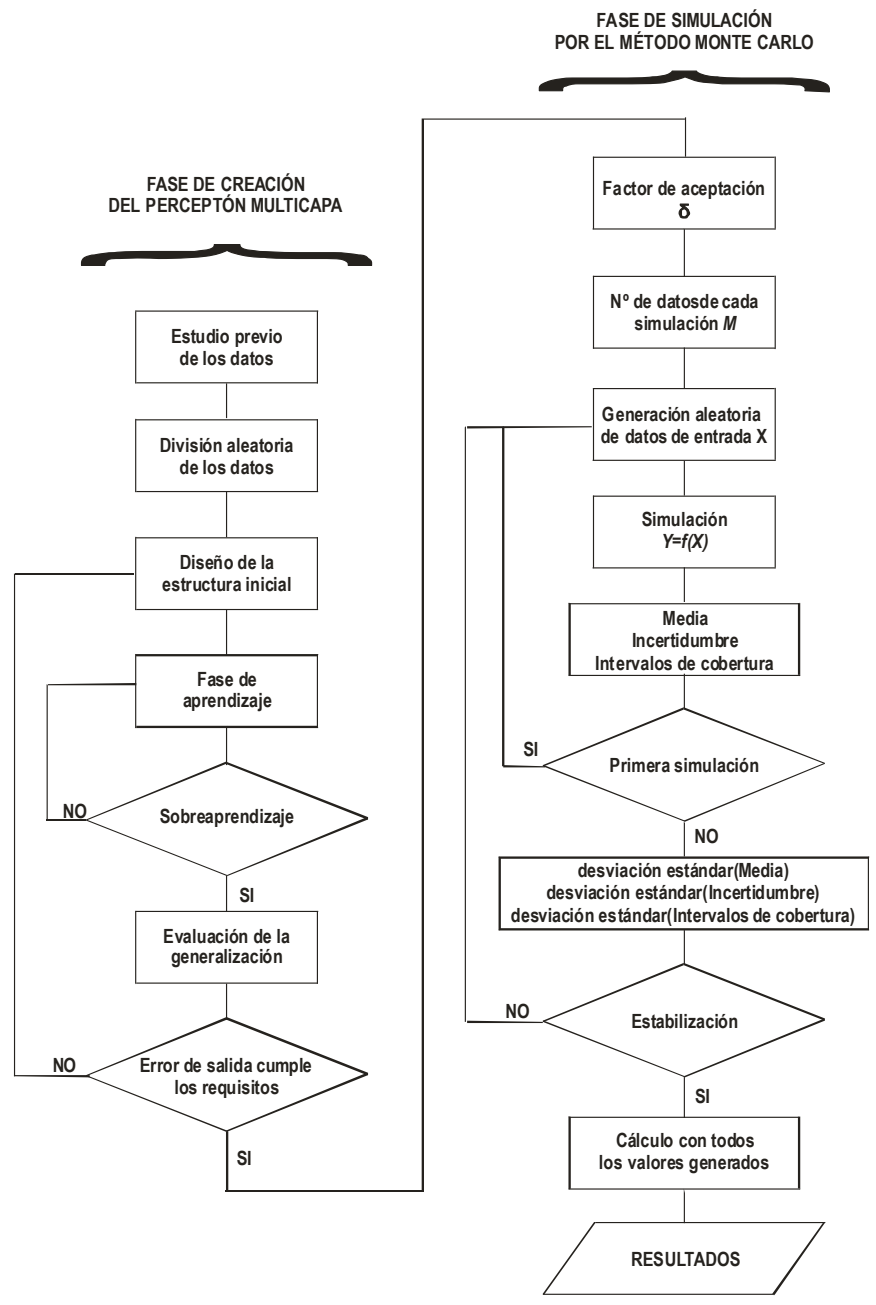

Fig. 2. Diagrama de flujo del método adaptativo de Montecarlo.

$$
\begin{gathered}
\operatorname{PICP}(\%)=100 \cdot \frac{1}{n_{\text {test }}} \sum_{1}^{n_{\text {test }}} c_{i} \\
c_{i}=\left\{\begin{array}{ll}
1 & t_{i} \in\left[L_{i}, U_{i}\right] \\
0 & t_{i} \notin\left(L_{i}, U_{i}\right)
\end{array}\right\}
\end{gathered}
$$

$n_{\text {test: }}$ número de datos experimentales; $L_{i}$ y $U_{i}$ : límites inferior y superior de los intervalos de confianza del valor $i$-ésimo; $t_{i}$ : i-ésimo valor experimental.

De acuerdo a [18] y [19] es de esperar que $P I C P(\%)$ supere el $95 \%$.

Para todos los cálculos se ha desarrollado un programa específico en lenguaje MATLAB®.

\section{Resultados}

\subsection{Incertidumbres de las variables de entrada}

Las incertidumbres de las variables de entrada y salida se han obtenido a partir de los datos de los ensayos.

Los valores obtenidos para cada uno de los parámetros de entrada, así como sus incertidumbres, se reflejan en las siguientes tablas (tablas 2 y 3 ).

Tabla 2. Variables de entrada.

\begin{tabular}{|c|c|c|c|}
\hline Variable & Media & $\begin{array}{c}\text { Desv. } \\
\text { típica }\end{array}$ & Incert. \\
\hline $\begin{array}{c}\text { Periodo de } \\
\text { curado (días) }\end{array}$ & 43.2 & 30.3 & 0.9 \\
\hline \% aditivo & 2.0 & 0.35 & $1.2 \cdot 10^{-2}$ \\
\hline Aditivo (kg/m3) & 12.7 & 3.8 & 0.7 \\
\hline \% Microsílice & 7.5 & 2.0 & $6.6 \cdot 10^{-2}$ \\
\hline $\begin{array}{c}\text { Microsílice } \\
\text { (kg/m3) }\end{array}$ & 48.1 & 15.0 & 0.8 \\
\hline a/c & 0.3 & 0.04 & $1.5 \cdot 10^{-3}$ \\
\hline $\begin{array}{c}\text { Cemento } \\
\text { (kg/m3) }\end{array}$ & 586.7 & 80.0 & 2.6 \\
\hline $\mathrm{TNM} \mathrm{(“)}$ & 1 & 0.06 & $2.2 \cdot 10^{-3}$ \\
\hline Arena (kg/m3) & 423.7 & 93.3 & 2.9 \\
\hline
\end{tabular}

Tabla 3. Constantes de entrada

\begin{tabular}{|c|c|c|}
\hline Parámetro & Valor & Incertidumbre \\
\hline Agua $(\mathrm{L} / \mathrm{m} 3)$ & 186.4 & 0.7 \\
\hline PE arena $($ Ton $/ \mathrm{m} 3)$ & 2.7 & $8.1 \cdot 10^{-4}$ \\
\hline Piedra $(\mathrm{kg} / \mathrm{m} 3)$ & 1006.2 & 0.7 \\
\hline PE Piedra $($ Ton $/ \mathrm{m} 3)$ & 2.7 & $8.1 \cdot 10^{-4}$ \\
\hline
\end{tabular}

\subsection{Red neuronal artificial.}

La arquitectura óptima para el preceptrón multicapa consta de dos capas ocultas con 6 y 1 neuronas cada una. Los resultados del proceso de entrenamiento, validación y comprobación pueden observarse en la siguiente tabla (tabla 4).

Tabla 4. Resultado del proceso de diseño de la red neuronal artificial.

\begin{tabular}{|c|c|c|c|c|}
\hline Fase & Estructura & $\mathbf{R}^{\mathbf{2}}$ & $\mathbf{R}$ & $\begin{array}{c}\text { Error } \\
\mathbf{( \% )}\end{array}$ \\
\hline Entrenamiento & \multirow{2}{*}{$\left.\begin{array}{llll}15 & 6 & 1 & 1\end{array}\right]$} & 0.81 & 0.90 & 4.3 \\
\cline { 1 - 3 } \cline { 4 - 6 } Validación & & 0.81 & 0.90 & 4.2 \\
\cline { 1 - 4 } Comprobación & & 0.80 & 0.89 & 4.2 \\
\hline
\end{tabular}

La figura 3 muestra las correlaciones entre los datos experimentales y los obtenidos por la red durante la fase de comprobación. 
Los valores obtenidos para los coeficientes de determinación para el conjunto de comprobación (tabla 4) son de 0.80 y 0.81 , lo cual indica que los modelos obtenidos son capaces de explicar el $80 \%$ de la variabilidad de las muestras.

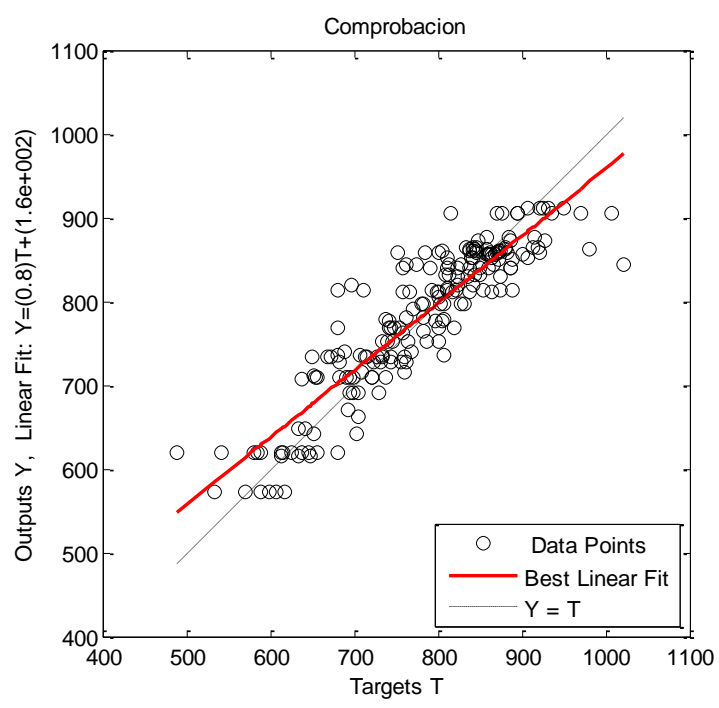

Fig. 3. Correlación entre los valores observados y predichos en el conjunto de comprobación

\subsection{Simulación de la incertidumbre mediante Montecarlo.}

Los resultados de la incertidumbre, obtenida mediante la simulación de Montecarlo, sobre los datos de los conjuntos de entrenamiento, validación y comprobación se muestran en la siguiente tabla (tabla 5).

Tabla 5. PICP sobre el conjunto de los datos

\begin{tabular}{|c|c|}
\hline Conjunto & $\boldsymbol{P I C P}(\%)$ \\
\hline Entrenamiento & 98.3 \\
\hline Validación & 98.2 \\
\hline Comprobación & 97.8 \\
\hline
\end{tabular}

\section{Discusión.}

Los resultados obtenidos para la red neuronal se encuentran dentro del rango de valores obtenidos en otros estudios de modelización de las propiedades del concreto. Así, los resultados obtenidos con coeficientes de correlación comprendidos entre 0.90 y 0.91 , son coherentes con los obtenidos por otros autores consultados [20 - 25], los cuales obtuvieron coeficientes de correlación entre 0.81 y 0.98 .
Igualmente los coeficientes de determinación obtenidos $\left(R^{2}=0.80\right.$ y $\left.R^{2}=0.81\right)$ son superiores a los obtenidos por [26] y similares a los obtenidos por [27] los cuales obtuvieron coeficientes máximos de 0.78 .

Los valores de PICP(\%) obtenidos son del $98 \%$ (tabla 6) indicando que casi todos los datos experimentales del estudio están incluidos dentro del intervalo de confianza. Se puede observar que el valor de $\operatorname{PICP}(\%)$ es superior al $95 \%$ indicado por [18] en su artículo y está dentro de los rangos obtenidos por [18], [1] o [17] los cuáles obtuvieron intervalos de confianza entre $75 \%$ y $100 \%$ dependiendo del método utilizado en la simulación.

El resultado es superior al obtenido por [10] en su estudio de los intervalos de confianza de las predicciones en los mercados de energía en el cual se obtuvieron $P I C P(\%)$ entre 92.6 y $94.1 \%$.

El resultado obtenido es también superior al obtenido por [28] en su estudio de los intervalos de confianza de las predicciones de demanda del mercado eléctrico, los cuáles obtuvieron PICP(\%) entre el $50 \%$ y el $100 \%$.

También es superior a los resultados reportados por [29] los cuales obtuvieron, para un factor de cobertura del $95 \%$, un $P I C P(\%)$ entre 89.6 y $99.6 \%$ dependiendo del método utilizado en la modelización. Y coherente con los resultados de [30] los cuales obtuvieron PICPs(\%) superiores al 95\% a partir de 50 simulaciones.

\section{Conclusiones.}

Se ha obtenido una red neuronal artificial con el suficiente nivel de confianza para ser utilizada como método alternativo al normalizado para la obtención de los resultados de resistencia a compresión del concreto de alta resistencia.

Se ha utilizado en método de Montecarlo para obtener la incertidumbre de los valores de salida de una red neuronal artificial obteniéndose unos niveles de confianza similares a otros estudios.

Por lo tanto, se ha comprobado la validez del uso de la técnica de Montecarlo para la simulación de la incertidumbre de los valores de resistencia a compresión obtenidos a partir de la simulación mediante una red neuronal artificial.

Se ha mejorado la posibilidad de la utilización de las redes neuronales artificiales para el control en fábrica de la resistencia a compresión ya que la incertidumbre del ensayo permite conocer el grado 
de cumplimiento/no cumplimiento de una especificación cuando los resultados obtenidos se encuentran próximos a los límites de la especificación.

\section{Agradecimientos}

Este trabajo se ha desarrollado se enmarca dentro del Proyecto de Investigación Proyecto PIAP-1-P676-13 financiado por FINCyT, dentro del Programa de Proyectos de Investigación aplicada 2013

\section{Referencias}

[1] A. Khosravi, S. Nahavandi, D. Creigton, A.F. Atiya, Lower upper bound estimation method for construction of neural networks-based prediction intervals. IEEE Transaction in Neural Networks. 22 (2011): 337-346.

[2] M. Cox, P. Harris, B.R.L. Siebert, Evaluation of measurement uncertainty based on the propagation of distributions using Monte Carlo simulation. Measurement Techniques. 46 (2003): 824-832.

[3] M. Solaguren-Beascoa Fernández, J.A. Alegre Calderón, P.M. Bravo Díez, Implementation in MATLAB of the adaptative Monte Carlo method for the evaluation of measurement uncertainties. Accreditation and Quality Assurance. 14 (2009): 95-106.

[4] BIMP, IEC, IFCC, ISO, IUPAC, OILM.. Guide to the Expression of Uncertainty in Measurement. 2nd ed. (International Organisation for Standardisation, Geneva, Switzerland, 1995)

[5] T.J. Esward, A. Ginestous, P.M. Harris, I.D. Hill, A Monte Carlo Method for uncertainty evaluation implemented on a distributed computing system. Metrologia. 44 (2007): 319326.

[6] M. Müler, M. Wolf, M. Rösslein, MUSE: computational aspects of a GUM supplement 1 implementation. Metrología. 45 (2008): 586594

[7] JCGM, Evaluation of measurement data . Supplement 1 to the "Guide to the expression of uncertainty in measurement" - Propagation of distributions using a Monte Carlo method. 1st ed. (Joint Commitee for Guides in Metrology, Sèvres, Francia, 2008).

[8] ASTM C 39/C 39M, Standard Test Method for Compressive Strength of Cylindrical Concrete Specimens. (The American Society for Testing Materials, West Conshohocken, PA, USA, 2001).

[9] ASTM C 192/C 192M, Standard Practice for
Making and Curing Concrete Test Specimens in the Laboratory. (The American Society for Testing Materials, West Conshohocken, PA, USA, 2000).

[10] A. Torre, F. García, I. Moromi, P. Espinoza, L. Acuña, Prediction of compression strength of high performance concrete using artificial neural networks. Journal of Physics: Conference Series. 582 (2015).

[11] L. Acuña, A. Torre, I. Moromi, F. García, Use of artificial neural networks to predict the compressive strength of concrete according to ASTM C39/C 39M standard. Información Tecnológica. 25 (2014): 3-12.

[12] L. Acuña, A. Torre, I. Moromi, P. Espinoza; F. García, Modelización de la Resistencia a la compresión del concreto mediante redes neuronales artificiales. TECNIA. 23 (2013): 1120.

[13] K. Hornik, M. Stinchcombe, H. White, Multilayer feedforward networks are universal approximators. Neural Networks. 2 (1989): 359-366

[14] W.Sha, Comment on the issues of statistical modelling with particular reference to the use of artificial neural networks. Applied Catalysis. A-General. 324 (2007): 87-89.

[15] B. Vanstone, G. Finnie, An empirical methodology for developing stockmarket Trading Systems using artificial neural Networks. Expert systems with Applications. 36 (2009): 6668-6680.

[16] H. Demuth, M. Beale, M. Hagan, Neural Network Toolbox User's Guide. Version 4. (The MathWorks Inc., Natick, USA, 2002).

[17] A. Khosravi, S. Nahavandi, D. Creigton, A.F. Atiya, Comprehensive review of neural network-based prediction intervals and new advances. IEEE Transaction in Neural Networks. 22 (2011): 1341-1356.

[18] E. Mazloumi, G. Rose, G. Currie, S. Moridpour, Prediction intervals to account for uncertainties in neural network predictions: Methodology and application in bus travel time prediction. Engineering Applications of Artificial Intelligence. 24 (2011): 534-542.

[19] A. Khosravi, S. Nahavandi, D. Creigton, Construction of optimal prediction intervals for load forecasting problems. IEEE Transaction on Power Systems. 25 (2010): 1496-1503.

[20] S. C. Lee, Prediction of concrete strength using artificial neural networks. Engineering Structures. 25 (2003): 849-57.

[21] A. Oztas, M. Pala, E. Ozbay, E. Kanka, A. Caglar, M. A. Bhatti, Predicting the compressive strength and slum of high 
strength concrete using neural network. Construction and Building Materials. 20 (2006): 769-75

[22] N. Ukrainczyk, V. Ukrainczyk, A neural network method for analysing concrete durability. Magazine of Concrete Research. 60 (2008): 475-86.

[23] M.S. Ozerdem, S. Kolukisa, Artificial neural network approach to predict the mechanical properties of $\mathrm{Cu}-\mathrm{Sn}-\mathrm{Pb}-\mathrm{Zn}-\mathrm{Ni}$ cast alloys. Materials and Design. 30 (2009): 764-769.

[24] B. K. R. Prasad, H. Eskandari, B. V. V. Reddy, Prediction of compressive strength of SCC and HPC with hay volumen fly ash using ANN. Construction and Building Materials. 23 (2009): 117-28.

[25] H. Yaprak, A. Karaci, I. Demir, Prediction of the effect of varying cure conditions and $\mathrm{w} / \mathrm{c}$ ratio on the compressive strength of concrete using artificial neural networks Neural Computing Applications. 22 (2013): 133-41.

[26] I. C. Yeh, Modeling of strength of highperformance concrete using artificial neural networks. Cement and Concrete Research. 28 (1998): 1797-808.
[27] M. Ozturan, B. Kutlu, T. Ozturan, Comparison of concrete strength prediction techniques with artificial neural network approach. Building Research Journal. 56 (2008): 23-36.

[28] N.A. Shrivastava, B.K. Panigrahi, Point and prediction interval estimation for electricity markets with machine learning techniques and wavelet transforms. Neurocomputing. 118 (2013): 301-310.

[29] C. Wan, Z. Xu, P. Pinson, Z.Y. Dong, K.P. Wong, Optimal prediction of wind power generation. IEEE Transactions on Power Systems. 29 (2014): 1166-1174.

[30] A. Khosravi, S. Nahavandi, An optimal mean variance estimation method for uncertainty quantification of wind power forecasts. Electric Power and Energy Systems. 61 (2014): 446454.

E-mail: francisco.garcia@upm.es, anatorre@uni.edu.pe, imoromi@yahoo.com, pcesp67@gmail.com, lacuna@uni.edu.pe 